\title{
Risco de quedas evidenciado por idosos atendidos num ambulatório de geriatria
}

Risk of falls displayed by elderly patients cared for by a geriatric outpatient clinic

Riesgo de caídas evidenciado por ancianos atendidos en un ambulatorio de geriatría

Maria das Graças Melo Fernandes ${ }^{1}$, Keylla Talitha Fernandes Barbosa ${ }^{2}$, Fabiana Maria Rodrigues Lopes de Oliveira ${ }^{3}$, Mayara Muniz Dias Rodrigues ${ }^{4}$, Kamyla Félix Oliveira dos Santos ${ }^{5}$

\footnotetext{
${ }^{1}$ Enfermeira, Doutora em Ciências da Saúde. Professora Associada da Universidade Federal da Paraíba (UFPB). João Pessoa, PB, Brasil. E-mail:

graacafernandes@hotmail.com.

${ }^{2}$ Enfermeira. Discente do Programa de Pós-Graduação em Enfermagem (PPGEnf), nível Mestrado, da UFPB. João Pessoa, PB, Brasil. E-mail: keyllafernandes@gmail.com.

3 Enfermeira. Discente do PPGEnf/UFPB, nível Mestrado. João Pessoa, PB, Brasil. E-mail: fabianarodriguesenf@yahoo.com.br.

${ }^{4}$ Acadêmica do curso de graduação em Enfermagem da UFPB. João Pessoa, PB, Brasil. E-mail: mayara_muniz_@hotmail.com.

${ }^{5}$ Enfermeira, Mestre em Enfermagem. Discente do PPGEnf/UFPB, nível Doutorado. João Pessoa, PB, Brasil. E-mail: kamylaoliveira@hotmail.com.
}

\section{RESUMO}

Objetivou-se identificar o risco de quedas em idosos em atendimento ambulatorial e a associação entre seus fatores. Trata-se de um estudo transversal envolvendo 120 idosos. Para coleta de dados, utilizou-se um instrumento contendo a avaliação sociodemográfica, clínica e o FallRisck Score (FRS). Os dados foram analisados com a utilização de estatística descritiva e o teste do Qui-quadrado. Os resultados encontrados demonstram que a ocorrência de quedas foi elevada em: $73,4 \%$ das mulheres; $61,2 \%$ dos sedentários; $60,8 \%$ daqueles na faixa etária de 60 a 69 anos; $55,8 \%$ dos hipertensos; e em $41,22 \%$ daqueles que usaram um a dois medicamentos. Dado o exposto, recomenda-se a reflexão, por parte dos serviços de saúde, sobretudo, quanto à atenção básica no que diz respeito à prevenção e identificação precoce dos fatores de riscos para quedas com o intuito de minimizar a elevada prevalência no que se refere a idosos que se encontram em âmbito de atendimento ambulatorial.

Descritores: Idoso; Fatores de Risco; Enfermagem Geriátrica.

\section{ABSTRACT}

This study aims to identify risk factors for falls among elderly patients cared for at an outpatient clinic and the association between such factors. It is a cross-sectional study with 120 elderly patients. Data was collected using an instrument which contained a sociodemographic and clinical evaluation and the FallRisk Score (FR). The data was analyzed using descriptive statistics and the chi-squared test. Results demonstrate that the occurrence of falls was elevated in: $73.4 \%$ of women; $61.2 \%$ of sedentary patients; $60.8 \%$ of those between 60 and 69 years of age; $55.8 \%$ of hypertensive patients and $41.22 \%$ of those taking one or two medications. Considering this information, we recommend that there be a reflection, especially from health services, on basic care regarding prevention and early identification of risk factors for falls, in order to minimize their elevated prevalence among elderly patients cared for at the outpatient clinic level.

Descriptors: Aged; Risk Factors; Geriatric Nursing.

\section{RESUMEN}

Se objetivó identificar riesgo de caídas en ancianos bajo atención ambulatoria y asociación entre sus factores. Estudio transversal, involucrando a 120 ancianos. Datos recolectados mediante instrumento conteniendo evaluación sociodemográfica, clínica y el FallRisk Score (FRS). Los datos fueron analizados utilizándose estadística descriptiva y test de Chi-cuadrado. Los resultados encontrados demuestran que la ocurrencia de caídas fue elevada en: $73,4 \%$ de mujeres; $61,2 \%$ de sedentarios; $60,8 \%$ de personas comprendidas en faja etaria de 60 a 69 años; 55,8\% de hipertensos, y en $41,22 \%$ de usuarios de uno a dos medicamentos. Según lo expuesto, se recomienda reflexión por parte de los servicios de salud, particularmente a la atención básica, en lo tocante a prevención e identificación precoz de factores de riesgo para caídas, apuntando a disminuir la alta prevalencia de los mismos en ancianos que se encuentran en el nivel de atención ambulatoria.

Descriptores: Anciano; Factores de Riesgo; Enfermería Geriátrica. 


\section{INTRODUÇÃO}

O envelhecimento populacional é tido como uma das mais importantes transições demográficas observadas a nível mundial, acontecendo de forma rápida e abrupta principalmente países em desenvolvimento, a exemplo do Brasil. Tal fato se deve aos avanços nas áreas de saúde, tecnologia e indústria farmacêutica, além da adoção de hábitos de vida saudáveis. A adoção de políticas públicas de saúde com o objetivo de promover a saúde e prevenir as doenças também têm contribuído para esse cenário(1). Caso seja mantida a atual dinâmica, a partir de 2030, o total de idosos ultrapassará o número de jovens entre 15 e 29 anos $^{(2)}$.

Ressalta-se que, associada à transição demográfica, verificou-se também mudança no perfil epidemiológico da população. O aumento na prevalência de doenças crônicas não transmissíveis (DCNT) é uma das principais características do processo de transição epidemiológica. A prevalência de algumas dessas doenças eleva-se a partir dos 60 anos, destacando-se: as doenças osteoarticulares, a hipertensão arterial sistêmica (HAS), as doenças cardiovasculares, o diabetes mellitus, as doenças respiratórias crônicas, a doença cerebrovascular e o câncer ${ }^{(3)}$.

Do ponto de visto biológico, o envelhecimento é um processo dinâmico e progressivo, envolvendo alterações morfológicas, funcionais, sociais e psicológicas, tornando-o mais suscetível às agressões intrínsecas e extrínsecas. Convém salientar que hábitos de vida interagem comumente com a genética, fazendo as consequências do envelhecimento variáveis de indivíduo para indivíduo. Destaca-se dentre as principais preocupações relacionadas à saúde advindas com a longevidade as ocorrências de doenças crônicas, quedas e incapacidade funcional, fazendo com que esses indivíduos necessitem, em muitos casos, de cuidado permanente por parte do sistema de saúde ${ }^{(4)}$.

Considerando-se particularmente o fenômeno queda, este pode ser definido como um deslocamento não intencional do corpo para um nível inferior à posição inicial com incapacidade de correção em tempo hábil, determinado por circunstâncias multifatoriais variáveis conforme a cultura e condições de vida dos idosos, comprometendo a estabilidade ${ }^{(5)}$.

Dados provenientes do Ministério da Saúde do Brasil evidencia que, entre os anos de 1996 e 2005, cerca de 24.645 idosos morreram devido a quedas, ocupando a terceira causa de mortalidade e o primeiro lugar dentre as internações hospitalares ${ }^{(6)}$. Além do risco aumentado para a morte, as quedas trazem outras consequências para os idosos, tais como: declínio ou perda da capacidade funcional, medo de sofrer novas quedas, e isolamento social. Destarte, a elevada incidência das quedas na população idosa e suas consequências acarretam elevados custos para o sistema de saúde e interferem na qualidade de vida do idoso, trazendo mudanças significativas à vida cotidiana(7).

Cabe destacar que apesar da relevância do problema, há dificuldades em se reconhecer o motivo das quedas, pois essas, conforme mencionado, têm origem multifatorial. Dentre os fatores de risco mais comuns, evidencia-se a idade avançada, sexo feminino, déficit de equilíbrio, distúrbios de marcha, baixa aptidão física, diminuição da força muscular, hipotensão postural, baixa acuidade visual, déficit cognitivo e polifarmácia ${ }^{(1,7)}$.

Nessa perspectiva, justifica-se a realização de estudos envolvendo a temática 'quedas', visto que esta possui um grande significado dentre a população idosa, dada a sua frequência elevada e caráter debilitante, podendo resultar em incapacidades, injúria e morte. Destarte, a prevenção das quedas deve constituir ponto primordial nos cuidados da Enfermagem gerontológica. Em contrapartida, percebe-se que tanto nas observações empíricas dos profissionais de saúde quanto nos resultados obtidos de pesquisas científicas realizadas em diferentes contextos, identifica-se ênfase nos estudos de prevalência no âmbito comunitário em detrimento daqueles que tenham como foco a mensuração dos fatores de risco em idosos em atendimento ambulatorial.

Diante desse contexto, o estudo pode subsidiar um aporte teórico para construção de uma proposta de cuidados, mediante o reconhecimento e quantificação dos riscos que envolvem o evento quedas. Assim sendo, o presente estudo teve como objetivo identificar o risco de quedas em idosos em atendimento ambulatorial e a associação entre seus fatores.

\section{METODOLOGIA}

Trata-se de um estudo transversal, descritivo, realizado dentre idosos atendidos em um ambulatório de geriatria de um hospital universitário do município de João Pessoa - PB. A amostra constitui-se de 120 idosos, de ambos os sexos, atendidos no referido ambulatório no período de outubro à dezembro de 2011 .

Quanto aos critérios de inclusão, fizeram parte do estudo idosos que aceitaram participar da pesquisa, com escores no Mini-exame do Estado Mental - MEEM $^{(8)}$ superiores a 13 (analfabetos) e a 17 (alfabetizados), sem comprometimento de fala e/ou audição que os impedissem de responder a entrevista. Foram excluídos 
do estudo aqueles que apresentaram déficit cognitivo moderado/acentuado, escores no MEEM inferiores a 13 (analfabetos) e a 17 (alfabetizados), os que tinham amputações e/ou uso de próteses em membros, aqueles que evidenciaram sequelas oriundas de acidente vascular encefálico, doença de Parkinson, fraturas em membros inferiores e/ou coluna, assim como os que faziam uso de cadeira de rodas.

A coleta de dados ocorreu mediante entrevista, sendo subsidiada por um instrumento estruturado que contemplava questões para avaliação das variáveis sociodemográficas, clínicas, medicamentos em uso contínuo e risco de quedas. Para avaliação do risco de quedas dentre os idosos foi utilizada a FallRisk Score (FRS), traduzida para a Língua Portuguesa e validada no Brasil $^{(9)}$.Essa escala mensura o risco de quedas a partir das seguintes variáveis: quedas anteriores, medicações que podem ocasionar o evento queda, déficit sensorial, estado mental e marcha. A pontuação do instrumento varia de zero a 11 , sendo que pontuação igual ou superior a três indica um alto risco para quedas.

A análise dos dados foi efetivada em uma abordagem quantitativa por meio de estatística descritiva e inferencial, utilizando-se sistema computacional StatisticalPackage for the Social Sciences - SPSS versão 20.0, por ser adequada ao alcance dos objetivos do estudo e por possibilitar a precisão e generalização dos seus resultados. Para a comparação dentre os grupos de variáveis foi utilizado o Teste do Qui-quadrado, sendo adotado nível de significância de 5\%.

O projeto foi apreciado e aprovado pelo Comitê de Ética do Hospital Universitário Lauro Wanderley (HULW), processo no 240/11 e CAAE 0129.0.126.000 - 11. Obedeceu-se às diretrizes e normas regulamentares de pesquisa que envolvem seres humanos, Resolução no 196 do Conselho Nacional de Saúde, de 10 de outubro de $1996^{(10)}$, vigente no período da pesquisa, sendo esta revogada pela Resolução no 466, de 12 de dezembro de $2012^{(11)}$.

\section{RESULTADOS}

Do total de 120 idosos, 95 (79,2\%) eram do sexo feminino e com faixa etária predominante entre 60 a 69 anos (60,8\%). Quanto ao estado civil, $57(47,5 \%)$ idosos eram casados e $109(90,8 \%)$ residiam com outros membros da família (Tabela 1 ).

A prevalência de quedas no último ano dentre os idosos foi de $40,83 \%$. As quedas foram mais comuns entre as mulheres $(73,47 \%)$ e nos indivíduos que não praticavam atividade física regularmente (61,22\%), conforme explícito na Tabela 2 . No que diz respeito ao alto risco para quedas, verifica-se que as mulheres apresentaram maior risco de cair $(79,75 \%)$ quando comparadas aos homens $(20,25 \%)$. Idosos entre 60 a 69 anos $(55,70 \%)$, casados $(49,37 \%)$ e que residiam com outros membros da família $(94,94 \%)$ apresentaram alto risco para quedas (Tabela 3). Ao se estabelecer associação entre o alto risco para quedas e doenças referidas pelos idosos, destacaram-se os seguintes problemas de saúde: hipertensão arterial (83,3\%), problemas de coluna $(82,5 \%)$, e visão prejudicada $(76,2 \%)$. Essa associação foi estatisticamente significativa entre: hipertensão arterial $(p<0,001)$, visão prejudicada $(p<0,001)$, reumatismo/artrose $(0,003)$, varizes $(p=0,012)$, depressão $(p=0,023)$ e audição prejudicada ( $p=0,011$ ). É oportuno lembrar que cada idoso poderia referir mais de um problema de saúde (Tabela 4).

Dentre os idosos, 114 (95\%) utilizavam regularmente medicamentos. Ao se estabelecer associação entre o uso de medicamentos e alto risco para quedas, verificou-se que entre os idosos que apresentavam esse risco, $33(41,2 \%)$ utilizavam um a dois medicamentos de forma regular e 15 (18,8\%) utilizavam cinco ou mais medicamentos de forma concomitante, caracterizando a polifarmácia. Verificou-se que $69(86,2 \%)$ idosos utilizavam anti-hipertensivos, 32 $(40 \%)$ diuréticos, $30(37,5 \%)$ tranquilizantes e 10 $(12,5 \%)$ antidepressivos (Tabela 5$)$

Tabela 1: Distribuição das características da amostra segundo as variáveis independentes ( $n=120)$. João Pessoa, PB, Brasil, 2012.

\begin{tabular}{|c|c|c|c|c|}
\hline Variável & Categorias & $\mathbf{N}$ & $\%$ & IC 95\% \\
\hline \multirow{2}{*}{ Sexo } & Feminino & 95 & $79,2 \%$ & $(70,9 \%-86,7 \%)$ \\
\hline & Masculino & 25 & $20,8 \%$ & $(13,3 \%-29,1 \%)$ \\
\hline \multirow{3}{*}{ Faixa etária } & $60-69$ & 73 & $60,8 \%$ & $(51,7 \%-70,0 \%)$ \\
\hline & $70-79$ & 36 & $30,0 \%$ & $(22,5 \%-39,2 \%)$ \\
\hline & Casado & 57 & $47,5 \%$ & $(38,4 \%-56,7 \%)$ \\
\hline \multirow[t]{2}{*}{ Estado civil } & Viúvo & 40 & $33,3 \%$ & $(24,2 \%-41,7 \%)$ \\
\hline & Separado & 12 & $10,0 \%$ & $(5,0 \%-15,8 \%)$ \\
\hline \multirow{2}{*}{ Residente } & Acompanhado & 109 & $90,8 \%$ & $(85 \%-95,8 \%)$ \\
\hline & Sozinho & 11 & $9,2 \%$ & $(4,2 \%-15 \%)$ \\
\hline
\end{tabular}


Tabela 2: Distribuição dos idosos que sofreram quedas no último ano $(n=49)^{*}$. João Pessoa, PB, Brasil, 2012.

\begin{tabular}{|c|c|c|c|c|c|c|c|}
\hline \multirow{3}{*}{ Variável } & \multicolumn{4}{|c|}{ Sexo } & \multirow{2}{*}{\multicolumn{2}{|c|}{ Total }} & \multirow{3}{*}{$\mathbf{P}$} \\
\hline & \multicolumn{2}{|c|}{ Feminino } & \multicolumn{2}{|c|}{ Masculino } & & & \\
\hline & $\mathbf{n}$ & $\%$ & $\mathbf{n}$ & $\%$ & $\mathbf{n}$ & $\%$ & \\
\hline Sofreu quedas no último ano? & & & & & & & 0,202 \\
\hline $\operatorname{Sim}$ & 36 & 73,47 & 13 & 26,53 & 49 & 40,83 & \\
\hline Pratica atividades físicas ${ }^{(a)}$ & & & & & & & 0,075 \\
\hline Sim & 13 & 36,11 & 6 & 46,15 & 19 & 38,78 & \\
\hline Não & 23 & 63,89 & 7 & 53,85 & 30 & 61,22 & \\
\hline
\end{tabular}

Tabela 3: Distribuição dos idosos que evidenciaram risco de quedas segundo as variáveis sociodemográficas e ocorrência de quedas no último ano $(n=120)$. João Pessoa, PB, Brasil, 2012.

\begin{tabular}{|c|c|c|c|c|c|c|c|}
\hline \multirow{3}{*}{ Variável } & \multicolumn{4}{|c|}{ Risco de Quedas } & \multirow{2}{*}{\multicolumn{2}{|c|}{ Total }} & \multirow{3}{*}{$\mathbf{P}$} \\
\hline & \multicolumn{2}{|c|}{ Alto risco } & \multicolumn{2}{|c|}{ Baixo risco } & & & \\
\hline & $\mathbf{n}$ & $\%$ & $\mathbf{n}$ & $\%$ & $\mathbf{n}$ & $\%$ & \\
\hline Sexo & & & & & & & 0,751 \\
\hline Feminino & 63 & 79,75 & 32 & 78,05 & 95 & 79,17 & \\
\hline Masculino & 16 & 20,25 & 9 & 21,95 & 25 & 20,83 & \\
\hline Faixa etária & & & & & & & 0,344 \\
\hline $60-69$ & 44 & 55,70 & 29 & 70,73 & 73 & 60,83 & \\
\hline $70-79$ & 27 & 34,18 & 9 & 21,95 & 36 & 30,00 & \\
\hline 80 ou mais & 8 & 10,13 & 3 & 7,32 & 11 & 9,17 & \\
\hline Estado civil & & & & & & & 0,489 \\
\hline Casado & 39 & 49,37 & 18 & 43,90 & 57 & 47,50 & \\
\hline Viúvo & 24 & 30,38 & 16 & 39,02 & 40 & 33,33 & \\
\hline Solteiro & 6 & 7,59 & 5 & 12,20 & 11 & 9,17 & \\
\hline Separado & 10 & 12,66 & 2 & 4,88 & 12 & 10,00 & \\
\hline
\end{tabular}

Tabela 4: Distribuição dos idosos que evidenciaram risco de quedas segundo

as principais morbidades referidas $(n=120)$. João Pessoa, PB, Brasil, 2012.

\begin{tabular}{|c|c|c|c|c|c|c|c|}
\hline \multirow{3}{*}{ Morbidade } & \multicolumn{4}{|c|}{ Risco de Quedas } & \multirow{2}{*}{\multicolumn{2}{|c|}{ Total }} & \multirow{3}{*}{$\mathbf{P}$} \\
\hline & \multicolumn{2}{|c|}{ Alto risco } & \multicolumn{2}{|c|}{ Baixo risco } & & & \\
\hline & $\mathbf{n}$ & $\%$ & $\mathbf{n}$ & $\%$ & $\mathbf{n}$ & $\%$ & \\
\hline Problemas de coluna & 66 & 55 & 28 & 23,3 & 94 & 12,89 & 0,117 \\
\hline Hipertensão & 67 & 55,8 & 16 & 13,3 & 83 & 11,98 & $<0,001$ \\
\hline Visão prejudicada & 61 & 50,8 & 12 & 10 & 73 & 10,01 & $<0,001$ \\
\hline Reumatismo/Artrose & 51 & 42,5 & 14 & 11,6 & 65 & 8,91 & 0,003 \\
\hline Varizes & 47 & 39,1 & 14 & 11,6 & 61 & 8,36 & 0,012 \\
\hline Labiritinte & 40 & 33,3 & 16 & 13,3 & 56 & 7,68 & 0,200 \\
\hline Osteoporose & 25 & 20,8 & 16 & 13,3 & 41 & 5,62 & 0,226 \\
\hline Incontinência Urinária & 31 & 25,8 & 9 & 7,5 & 40 & 5,48 & 0,056 \\
\hline Cardiopatia & 28 & 23,3 & 10 & 10 & 38 & 5,21 & 0,184 \\
\hline Depressão & 27 & 22,5 & 6 & 5 & 33 & 4,52 & 0,023 \\
\hline Diabetes & 24 & 20 & 6 & 5 & 30 & 4,11 & 0,056 \\
\hline Audição prejudicada & 24 & 20 & 4 & 3 & 28 & 3,84 & 0,011 \\
\hline Total de Respostas & 491 & 100 & 174 & 151 & 729 & 100 & \\
\hline
\end{tabular}

* A soma perfaz valor superior a amostra em decorrência de um mesmo idoso apresentar mais de um problema de saúde.

Tabela 5: Distribuição dos idosos que evidenciaram risco de quedas segundo a quantidade e classes de medicamentos utilizados diariamente $(n=120)$. João Pessoa, PB, Brasil, 2012.

\begin{tabular}{|c|c|c|c|c|c|c|c|}
\hline \multirow{3}{*}{ Variável } & \multicolumn{4}{|c|}{ Risco de quedas } & \multirow{2}{*}{\multicolumn{2}{|c|}{ Total }} & \multirow{3}{*}{$\mathbf{P}$} \\
\hline & \multicolumn{2}{|c|}{ Alto risco } & \multicolumn{2}{|c|}{ Baixo risco } & & & \\
\hline & $\mathbf{n}$ & $\%$ & $\mathbf{n}$ & $\%$ & $\mathbf{n}$ & $\%$ & \\
\hline Medicamentos & & & & & & & 0,003 \\
\hline Nenhum & 1 & 1,2 & 5 & 12,5 & 6 & 5 & \\
\hline 1 a 2 & 33 & 41,2 & 24 & 60 & 57 & 47,5 & \\
\hline 3 a 4 & 31 & 38,8 & 6 & 15 & 37 & 30,8 & \\
\hline 5 ou mais & 15 & 18,8 & 5 & 12,5 & 20 & 16,7 & \\
\hline Total & 80 & 100 & 40 & 100 & 120 & 100 & \\
\hline Anti-hipertensivos & & & & & & & $<0,001$ \\
\hline Sim & 69 & 86,2 & 13 & 32,5 & 82 & 68,3 & \\
\hline Não & 11 & 13,8 & 27 & 67,4 & 38 & 31,7 & \\
\hline Diuréticos & & & & & & & $<0,001$ \\
\hline Sim & 32 & 40 & 2 & 15 & 34 & 28,3 & \\
\hline Não & 48 & 60 & 38 & 95 & 86 & 71,7 & \\
\hline Tranquilizantes & & & & & & & 0,011 \\
\hline Sim & 30 & 37,5 & 6 & 15 & 36 & 30 & \\
\hline Não & 50 & 62,5 & 34 & 85 & 84 & 70 & \\
\hline Antidepressivos & & & & & & & 0,020 \\
\hline Sim & 10 & 12,5 & 0 & 0 & 10 & 8,3 & \\
\hline Não & 70 & 87,5 & 40 & 100 & 110 & 91,7 & \\
\hline
\end{tabular}




\section{DISCUSSÃO}

Considerando os dados sociodemográficos evidenciados na Tabela 1 , verificou-se predomínio de $79,2 \%$ de idosos do sexo feminino. Em consonância com este achado, pesquisa realizada com 401 idosos no munícipio de João Pessoa evidenciou que, dentre eles, $76,6 \%$ eram mulheres, confirmando a tendência da feminização na velhice ${ }^{(12)}$. Ressalta-se que tal dado assemelha-se também aos achados de outros estudos realizados no Brasil nos quais se verificou a maior expectativa de vida dentre as mulheres, a qual está associada a fatores como: menor consumo de álcool e/ ou tabaco e, diferenças de atitude em relação às patologias $^{(5)}$.

No que se refere à faixa etária, o presente estudo obteve um expressivo percentual de idosos entre 60 a 69 anos, representando 60,8\%. Similarmente, pesquisa realizada com 275 idosos verificou que 45,5\% estavam nessa faixa etária ${ }^{(13)}$.A proporção de idosos que eram casados e residiam com outros membros da família foi respectivamente $47,5 \%$ e $90,8 \%$. Estudo realizado com 105 idosos corrobora tais achados, no qual identificou-se que $54,7 \%$ dos idosos eram casados e $91,3 \%$ residiam com outros membros da família(14).

A ocorrência de quedas no último ano na população estudada foi de 40,83\%, valor semelhante ao evidenciado em pesquisa realizada com idosos residentes em comunidade, dentre os quais 59,3\% apresentaram ao menos um episódio de quedas ${ }^{(8)}$. Prevalências inferiores foram relatadas na literatura nacional ${ }^{(15)}$, contudo, as diferenças podem ser atribuídas ao delineamento dos estudos e as diferentes características das populações estudas.

Dentre os idosos que sofreram quedas, $61,22 \%$ afirmaram não praticar atividades físicas regularmente, valor ínfero ao observado em estudo realizado com 96 idosos do município de Amparo, Estado de São Paulo, no qual se identificou que $76 \%$ dos idosos que sofreram quedas relataram não praticar nenhuma atividade física $^{(16)}$. Quando o idoso sofre uma queda há diminuição da autoconfiança, o que pode acarretar em um maior nível de inatividade. Dessa forma, o sedentarismo aumenta o risco de quedas no idoso, limita sua independência nas atividades de vida diária e convívio social. Pesquisas atribuem à realização de exercícios físicos regulares a capacidade de manter um bom desempenho funcional, de prevenir distúrbios da marcha e do equilíbrio em idosos ${ }^{(17)}$.

No que diz respeito ao risco para quedas, este estudo verificou que $79,75 \%$ das mulheres entrevistadas apresentavam alto risco de cair. Este resultado mostrase consistente se comparado com achados na literatura científica, destacando a prevalência de quedas no sexo feminino(15,18-19). Contudo, ainda não há uma explicação conclusiva sobre este fato, o qual parece estar relacionado ao menor estado funcional, maior morbidade e ocorrência de artrose. Outro fator importante é a redução do estrógeno a partir dos quarenta anos de idade, onde há perda de massa óssea que pode contribuir para deteriorar o estado funcional. Além das alterações fisiológicas próprias do envelhecimento, verifica-se também que os fatores pessoais e ambientais aumentam o risco para as quedas entre as mulheres ${ }^{(20)}$.

Pesquisas apontam que quanto maior a idade do idoso, maior é o risco de cair, visto que a idade avançada está intimamente ligada aos fatores de exposição a quedas que se acumulam de maneira progressiva $(1,18,21)$.Entretanto, o presente estudo verificou que $55,70 \%$ dos idosos que evidenciavam alto risco para quedas pertenciam a faixa etária de 60 a 69 anos. Isto pode está associado a menor deambulação dos idosos com maior idade, provavelmente por não possuírem força suficiente nos membros inferiores. Estudo demonstra que a força muscular declina com a idade e, sobretudo, com a idade mais avançada, uma vez que há perda gradual de $10 \%$ por década a partir dos 50 $\operatorname{anos}^{(22)}$. Esses achados podem ainda ser motivados pela justificativa de que a população estudada se reporta a idosos atendidos a nível ambulatorial, onde houve predominância de idosos jovens.

No que concerne o estado civil, $49,37 \%$ dos idosos que possuíam alto risco para quedas eram casados. Semelhantemente, estudo realizado com 130 idosos adscritos na Estratégia Saúde da Família (ESF) do município de João Pessoa, o qual verificou que 46,6\% dos idosos com risco para quedas viviam com 0 cônjuge ${ }^{(14)} . E m$ contrapartida, pesquisa demonstra a estreita relação entre viuvez e risco de cair ${ }^{(19)}$. O fato de ser viúvo não implica apenas em não ter companheiro, mas também em perdas sociais, podendo ser sinônimo de dependência física, econômica e afetiva, tornando o idoso mais suscetível à ocorrência de quedas ${ }^{(8,14)}$.

Dentre os idosos com alto risco para quedas, houve uma ocorrência significativa de idosos que referiram hipertensão arterial, representando 55,8\%. Corroborando com esse achado, estudo realizado com 95 idosos institucionalizados do município de Goiânia, Goiás, evidenciou que a maioria $(59,57 \%)$ refere problemas relacionados à pressão ${ }^{(20)}$.A hipertensão arterial sistêmica é a doença crônica mais comum dentre os 
idosos, sua prevalência aumenta progressivamente com a idade e consiste no principal fator de risco para os acidentes vasculares cerebrais. Alguns estudos apontam a história pregressa de acidente vascular cerebral como fator associado a quedas. Por outro lado, a hipotensão ortostática torna os idosos mais vulneráveis a quedas, por comprometer seu controle postural ${ }^{(23)}$.

Verificou-se que $55 \%$ dos idosos que detém alto risco para quedas referiram problemas de coluna. Em consonância com tal resultado, estudo realizado com 243 idosos do município de Pelotas (RS), evidenciou que doenças da coluna estavam presente em $42,7 \%$ dos idosos $^{(23)}$.Alguns autores apontam que as doenças relacionadas à coluna vertebral são frequentes na terceira idade e contribuem para redução da capacidade física, ocasionando dor, influenciando no equilíbrio ou controle postural, favorecendo a ocorrência de quedas ${ }^{(23-}$ 24).

Inúmeras alterações fisiológicas relacionadas à idade podem afetar o desempenho visual e levar ao risco de cair. O presente estudo verificou que $50,8 \%$ dos idosos com alto risco para quedas referiram problemas relacionados à visão. Os déficits de acuidade visual, restrição do campo visual, aumento da suscetibilidade à luz, percepção de profundidade deficiente ou instabilidade na fixação do olhar podem interferir no mecanismo de controle postural. Pesquisas demonstram que os idosos com leve e/ ou severa diminuição na função visual apresentaram, respectivamente, 1,4 e 2,2 vezes mais riscos de quedas ${ }^{(20,24)}$.

A frequência de eventos adversos relacionados aos medicamentos é maior dentre a população idosa, devido às alterações fisiológicas que interferem diretamente nos processos de absorção, distribuição, metabolização e eliminação dos fármacos. O risco de reações adversas aumenta de três a quatro vezes em pacientes submetidos a polifarmácia, podendo simular síndromes geriátricas ou precipitar quadros de confusão, incontinências e quedas ${ }^{(24)}$.Revela-se, então, como mais um risco para a população estudada, uma vez que o uso regular de medicamentos esteve presente em $95 \%$ dos idosos. Do mesmo modo, estudo realizado com 95 idosos do município de Goiânia verificou que o uso regular de medicações prescritas por médicos era feito por $91,6 \%$ deles $^{(24)}$.No presente estudo, $41,2 \%$ dos idosos com alto risco para quedas faziam uso de um a dois fármacos regularmente, corroborando com pesquisa semelhante ${ }^{(22)}$.

Dentre os idosos com alto risco de cair, $86,2 \%$ faziam uso de drogas anti-hipertensivas. Em conformidade com esse achado, estudo realizado com 95 idosos institucionalizados verificou que a classe de medicamento mais utilizada pelos idosos era a dos antihipertensivos, com $67,81 \%^{(23)}$. Os medicamentos cardiovasculares envolvem a predisposição de quedas em idosos em virtude dos efeitos colaterais como bradicardia, hipotensão, sonolência e fadiga ${ }^{(20)}$.Verificase no presente estudo que $40 \%$ dos idosos com alto risco para quedas utilizavam regularmente diuréticos; essas substâncias promovem depleção de volume e distúrbios hidroeletrolíticos, embora possa haver efeito protetor para quedas devido à redução na excreção urinária de cálcio e consequente aumento da densidade óssea ${ }^{(20,24)}$.

Ainda no tocante ao uso de medicamentos, verificouse que $37,5 \%$ dos idosos com alto risco para quedas faziam uso de tranquilizantes e $12,5 \%$ utilizavam antidepressivos regularmente. Com respeito a essas classes de medicamentos, verifica-se que os tranquilizantes e os antidepressivos podem causar sedação, alterações psicomotoras, relaxamento muscular e bloqueio beta-adrenérgico, aumentando a ocorrência de hipotensão ortostática, que por sua vez, aumenta a propensão para quedas ${ }^{(20)}$. A alta prevalência de medicação nos idosos desse estudo evidencia a necessidade de medidas preventivas quanto à associação da polifarmácia e o risco para quedas.

\section{CONCLUSÃo}

Os resultados encontrados demonstram que a ocorrência de quedas no último ano dentre os idosos investigados foi elevada, sendo mais frequente em mulheres que não praticam atividade física. Dentre os idosos que apresentam alto risco de cair, podemos identificar o seguinte perfil: mulheres, com idade entre 60 a 69 anos, casadas e que relatam morbidades como hipertensão arterial, problemas de coluna, visão prejudicada e utilizam de um a dois medicamentos regularmente, a exemplo, dos anti-hipertensivos.

Dentre as limitações deste estudo, ressalta-se o número amostral reduzido e por refletir apenas uma realidade local. Apesar desses entraves, observa-se que os resultados dessa pesquisa são relevantes à medida que contribuem para uma reflexão por parte dos serviços de saúde, sobretudo, na atenção básica no que diz respeito à prevenção e identificação precoce dos fatores de riscos para quedas, no intuito de minimizar a elevada prevalência desses nos idosos que se encontram em nível de atendimento ambulatorial. Recomenda-se assim a investigação do risco para quedas em outros cenários 
com a finalidade de verificar a associação desses fatores com o evento.

A identificação dos fatores associados ao risco para quedas é de grande importância para que se possa traçar métodos preventivos, detectar e acompanhar precocemente as incapacidades instaladas, assim como prevenir as quedas e, consequentemente, os danos

\section{REFERÊNCIAS}

1. Chianca TCM, Andrade CR, Albuquerque J, Wenceslau LCC, Tadeu LFR, Macieira TGR et al . Prevalência de quedas em idosos cadastrados em um Centro de Saúde de Belo Horizonte-MG. Rev Bras Enferm [Internet]. 2013 [acesso em: 30 jun

2014];66(2):234-40. Disponível em: http://dx.doi.org/10.1590/S0034-71672013000200013. 2. Instituto Brasileiro de Geografia e Estatística [Internet]. Brasília: Ministério do Planejamento, Orçamento e Gestão (BR); 2011 [acesso em: 30 jun 2014]. Sinopse do Censo Demográfico de 2010. Disponível em:

http://www.censo2010.ibge.gov.br/sinopse/.

3. Campolina AG, Adami F, Santos JLF, Lebrão ML. A transição de saúde e as mudanças na expectativa de vida saudável da população idosa: possíveis impactos da prevenção de doenças crônicas. Cad Saude Publica [Internet]. 2013 [acesso em: 30 jun 2014];29(6):1217-29. Disponível em:

http://dx.doi.org/10.1590/S0102-311X2013000600018. 4. Del Duca GF, Thume E, Hallal PC. Prevalence and factors associated with home care among older adults. Rev Saude Publica [Internet]. 2011 [acesso em: 30 jun 2014];45(1):11321. Disponível em: http://dx.doi.org/10.1590/S003489102010005000047.

5. Ramos CV, Santos SSC, Barlem ELD, Pelzer MT. Quedas em idosos de dois serviços de pronto atendimento do Rio Grande do Sul. Rev. Eletr. Enf. [Internet]. 2011 [acesso em: 30 jun 2014];13(4):703-13. Disponível em: http://dx.doi.org/10.5216/ree.v13i4.10856.

6. Alves EDF. Treinamento PREV-Quedas. Rio de Janeiro: SESIRJ; 2009.

7. Rezende AAB, Silva IL, Cardoso FB, Beresford H. Medo do idoso em sofrer quedas recorrentes: a marcha como fator determinante da independência funcional. Acta fisiátrica [Internet]. 2010 [acesso em: 30 jun 2014];17(3):117-21, Disponível em:

http://www.actafisiatrica.org.br/detalhe_artigo.asp?id=47.

8. Bertolucci PHF, Brucki SMD, Campacci SR, Juliano Y. O MiniExame do Estado Mental em uma população geral: impacto da escolaridade. Arq Neuropsiquiatr [Internet]. 1994 [acesso em: 30 jun 2014];52(1):1-7. Disponível em:

http://dx.doi.org/10.1590/S0004-282X1994000100001. 9. Schiaveto FV. Avaliação do risco de quedas em idosos na comunidade [dissertação]. São Paulo: Universidade de São Paulo; 2008.

10. Resolução No 466 do Conselho Nacional de Saúde, de 10 de outubro de 1996 (BR). Aprova as diretrizes e normas regulamentadoras de pesquisas envolvendo seres humanos. Diário Oficial da União. 16 out. 1996.

11. Resolução No 466 do Conselho Nacional de Saúde, de 12 de dezembro de 2012 (BR). Aprova as diretrizes e normas regulamentadoras de pesquisas envolvendo seres humanos. Diário Oficial da União. 13 jun. 2013.

12. Brito GEG, Silva AC, Oliveira FMRL, Barbosa KTF, Sá LR. Perfil dos idosos com cuidadores familiares adscritos à Estratégia Saúde da Família. Rev enferm UFPE on line. [Internet]. 2012 [acesso em: 30 jun 2014];6(7):1539-48. Disponível em: http://www.revista.ufpe.br/revistaenfermagem/index.php/revista /article/view/2725.

13. Ursine PGS, Cordeiro HA, Moraes CL. Prevalência de idosos restritos ao domicílio em região metropolitana de Belo Horizonte (Minas Gerais, Brasil). Cien Saude Colet [Internet]. 2011 [acesso físicos, internações e hospitalizações. Com o acentuado crescimento da população idosa e as dificuldades enfrentadas por ela em relação às condições de saúde, ressalta-se a necessidade de se discutir e implementar intervenções voltadas para a melhoria da sua qualidade de vida.

em: 30 jun 2014];16(6):2953-62. Disponível em:

http://dx.doi.org/10.1590/S1413-81232011000600033. 14. Pinho TAM, Silva AO, Tura LFR, Moreira MASP, Gurgel SN et al. Avaliação do risco de quedas em idosos atendidos em Unidade Básica de Saúde. Rev Esc Enferm USP [Internet]. 2012 [acesso em: 30 jun 2014];46(2):320-7. Disponível em: http://dx.doi.org/10.1590/S0080-62342012000200008. 15. Siqueira FV, Facchini LA, Silveira DS, Piccini RX, Tomasi E, Thumé $\mathrm{E}$ et al. Prevalence of falls in elderly in Brazil: a countrywide analysis. Cad Saude Publica [Internet]. 2011 [acesso em: 30 jun 2014];27(9):1819-26. Disponível em: http://dx.doi.org/10.1590/S0102-311X2011000900015. 16. Ricci NA, Gonçalves DFF, Coimbra IB, Coimbra AMV. Fatores associados ao histórico de quedas de idosos assistidos pelo Programa de Saúde da Família. Saúde Soc. [Internet]. 2010 [acesso em: 30 jun 2014];19(4):898-909. Disponível em: http://dx.doi.org/10.1590/S0104-12902010000400016. 17. Fernandes AMBL, Ferreira JJA, Stolt LROG, Brito GEG, Clementino ACCR, Sousa NM. Efeitos da prática de exercício físico sobre o desempenho da marcha e da mobilidade funcional em idosos. Fisioter. mov. [Internet]. 2012 [acesso em: 30 jun 2014];25(4):821-30. Disponível em:

http://dx.doi.org/10.1590/S0103-51502012000400015. 18. Cruz DT, Ribeiro LC, Vieira MT, Teixeira MTB, Bastos RR, Leite ICG. Prevalence of falls and associated factors in elderly individuals. Rev Saude Publica [Internet]. 2012 [acesso em: 30 jun 2014];46(1):138-46. Disponível em: http://dx.doi.org/10.1590/S0034-89102011005000087. 19. Motta LB, Aguiar AC, Coutinho ESF, Huf G. Prevalência e fatores associados a quedas em idosos em um município do Rio de Janeiro. Rev. Bras. Geriatr. Gerontol. [Internet]. 2010 [acesso em: 30 jun 2014];13(1):83-91. Disponível em:

http://revista.unati.uerj.br/scielo.php?script=sci_arttext\&pid=S1 809-98232010000100009\&lng=pt\&nrm=iso.

20. Ferreira DCO, Yoshitome AY. Prevalência e caraterísticas das quedas de idosos institucionalizados. Rev Bras Enferm [Internet]. 2010 [acesso em: 30 jun 2014];63(6):991-7. Disponível em: http://dx.doi.org/10.1590/S0034-71672010000600019.

21. Rodrigues J, Ciosak SI. Elderly victims of trauma: analysis of the risk factors. Rev Esc Enferm USP [Internet]. 2012 [acesso em: 30 jun 2014];46(6):1401-6. Disponível em: http://dx.doi.org/10.1590/S0080-62342012000600017. 22. Coutinho ES, Bloch KV, Rodrigues LC. Characteristics and circumstances of falls leading to severe fractures in elderly people in Rio de Janeiro, Brazil. Cad Saude Publica [Internet]. 2009 [acesso em: 30 jun 2014];25(2):455-9. Disponível em: http://dx.doi.org/10.1590/S0102-311X2009000200024. 23. Alvares LM, Lima RC, Silva RA. Ocorrência de quedas em idosos residentes em instituições de longa permanência em Pelotas, Rio Grande do Sul, Brasil. Cad Saude Publica [Internet]. 2010 [acesso em: 30 jun 2014];26(1):31-40. Disponível em: http://dx.doi.org/10.1590/S0102-311X2010000100004. 24. Secoli SR. Polifarmácia: interações e reações adversas no uso de medicamentos por idosos. Rev Bras Enferm [Internet]. 2010 [acesso em: 30 jun 2014];63(1):136-40. Disponível em: http://dx.doi.org/10.1590/S0034-71672010000100023.

Artigo recebido em 04/10/2012.

Aprovado para publicação em 31/01/2014.

Artigo publicado em 30/06/2014. 